

\section{Linha Editorial}

CAMPOS - Revista de Antropologia, publicação do Programa de Pós- Graduação em Antropologia da

Universidade Federal do Paraná, tem o propósito de constituir um espaço permanente de interlocução com antropólogos e pesquisadores de áreas afins, no país e no exterior. O nome CAMPOS traduz o reconhecimento do valor singular da etnografia para a Antropologia, remetendo também à pluralidade de perspectivas teóricas e temáticas que caracteriza a disciplina e que se revela na produção do próprio PPGA-UFPR. A Revista CAMPOS publica artigos inéditos, ensaios bibliográficos, entrevistas, resenhas e outras contribuições - pequenos textos de natureza acadêmica, informativa etc. - que podem ser enviados em português, inglês, francês ou espanhol (para publicação na língua original).

\section{Editoria}

Edilene Coffaci de Lima

\section{Comissão Editorial (PPGA-UFPR)}

Ciméa Barbato Bevilaqua Edilene Coffaci de Lima Eva Lenita Scheliga

Fábio Parenti

João Frederico Rickli Laércio Loiola Brochier Laura Pérez Gil

Liliana Mendonça Porto

Lorenzo Macagno

Marcos Silva da Silveira

Maria Inês Smiljanic

Miguel Alfredo Carid Naveira

Paulo Renato Guérios

Ricardo Cid Fernandes

Sandra Stoll

\section{Conselho Editorial}

América Larraín, Universidad Nacional de Colombia, Colômbia

Aristóteles Barcelos Neto,

University of East Anglia, Reino Unido

Birgit Meyer, Utrecht University, Holanda

Christiano Tambascia, UNICAMP, Brasil

Claudia Fonseca, UfRGS, Brasil

Eduardo Viveiros de Castro, MN/ URJ, Brasil

Elena Calvo Gonzalez, UFBA, Brasil

François Laplantine, Université

Lumière - Lyon 2, França

José Guilherme Cantor Magnani, USP, Brasil

Lorena Cordoba, CONICET/

Universidad de Buenos

Aires, Argentina e Centro de Investigaciones Históricas y Antropológicas, Bolivia

Manuela Carneiro da Cunha, (University of Chicago, EUA

Márcio Ferreira da Silva, USP, Brasil

Marcio Goldman, MN/UFRJ, Brasil

Martina Ahlert, UFMA, Brasil

Mariza G. S. Peirano, UnB, Brasil

Miriam P. Grossi, UfSC, Brasil

Peter Fry, UFRJ, Brasil

Philippe Erikson, Université de Paris Ouest-Nanterre, França

Rafael José Menezes Bastos, UFSC, Brasil

Roberto DaMatta, UFF/University of Notre Dame, Brasil/EUA

Roque de Barros Laraia, UnB, Brasil

Ruben G. Oliven, UfRGS, Brasil 


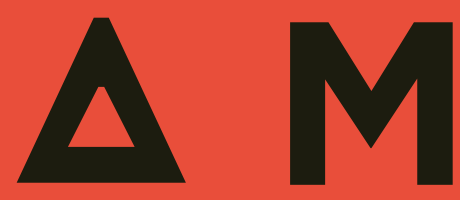

CAMPOS. Revista de Antropologia. PPGA/ UFPR.

V.19 N.1 janeiro/junho 2018 *Publicada em dezembro 2018 Curitiba, PR, Brasil ISSN 2317-6830

P. $001-216$

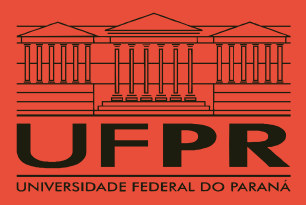


Qualquer parte desta publicação pode ser reproduzida, desde que citada a fonte.

Disponível também em:

revistas.ufpr.br/campos

Publicação indexada em:

Directory of Open Access Journals

- DOAJ (WwW.doaj.org)

Clase - Citas Latinoamericanas en

Ciencias Sociales y Humanidades

(http//132.248.9.1:8991)

Sumários de Revistas Brasileiras

(www.sumarios.org)

Latindex (www.latindex.unam.mx)

\section{Expediente}

\section{Secretaria de Redação}

Edilene Coffaci de Lima

\section{Foto da capa}

Criança no curral

Christine Escallier

\section{Revisão}

Edilene Coffaci de Lima

Programa de Apoio às Publicações

Científicas

\section{Preparação dos originais}

Edilene Coffaci de Lima

\section{Projeto gráfico}

Martim Fernandes

\section{Diagramação}

Juliano Buiarski de Oliveira

\section{Redação}

CAMPOS - Revista de Antropologia

Rua General Carneiro, 460 - $6^{\circ}$ andar. CEP 80.060-150 Curitiba Paraná - Brasil

Telefone: (41) 3360-5272

e-mail: revistacampos@gmail.com

CAMPOS - Revista de Antropologia Rua General Carneiro, 460 - $6^{\circ}$ andar. CEP 80.060-150 Curitiba - Paraná - Brasil

Telefone: (41) 3360-5272

revistacampos.ufpr@gmail.com

CAMPOS: Revista de Antropologia / Universidade Federal do Paraná. Programa de Pós-Graduação em Antropologia ; editores:

Ciméa Barbato Bevilaqua [...et al.], n.1 (2001) - Curitiba : UFPR/PPGAS, 2001.

n.19 (1), 2018 Semestral ISSN : $2317-6830$

1.Antropologia Social - Periódicos. I. Universidade Federal do Paraná. Programa de Pós-Graduação em Antropologia Social. II. Bevilaqua, Ciméa Barbato. 


\section{Dossiês}

Ecologia e Feminismo: criações políticas de mulheres indígenas, quilombolas e camponesas Fabiana Maizza e Suzane de Alencar Vieira

Sentir as palavras, criar trincheiras: mulheres em defesa da Red de la Vida Luiza Dias Flores \& Damiana Bregalda Jaenisch

Noke Mevi Revõsho Shovima Awe: o que é transformado pelas pontas das nossas mãos Nelly Barbosa Duarte Dollis

A morada é uma curandeira: o feminismo enquanto força

Luiza Dias Flores

Dos corpos, a terra: notas sobre a criatividade no trabalho de Rosa Tisoy Tatiana Lotierzo

o mundo dos projetos socioambientais visto pelas mulheres indígenas. Para repensar o ecofeminismo com o caso dos M bêngôkre -Xikrin da Terra Indígena Trincheira Bacajá (TITB, Pará, Brasil)

Stéphanie Tselouiko

\section{Artigos}

Mana e substâncias xamânicas: as reconfigurações de poder entre os Siona da Colômbia Esther Jean Langdon 
Mer, rivière, mangrove : les espaces marqués par le genre dans les communautés de pêche du Pará, Brésil

Christine Escallier

\section{Traduções}

163

Tradição e conhecimento, ou a vida ao ar livre - apresentação à tradução

Daniel Belik e Mariana Ciavatta Pantoja

Percebendo o ambiente na Lapônia finlandesa Tim Ingold e Terhi Kurttila

\section{Textos Informativos}

Yubaka Hayrá: Notas sobre a Conferência Indígena da Ayahuasca

Domingos Bueno da Silva

\section{Resenhas}

BASINI, José. 2015. Índios num país sem índios. A estética do desaparecimento: um estudo sobre imagens indias e versões étnicas. Manaus: Editora Travessia/Fapeam. 520pp. João Roberto Bort Júnior

BEVILAQUA, C.B.; VANDER VELDEN, F. (orgs.). 2016. Parentes, vítimas, sujeitos: perspectivas antropológicas sobre relações entre humanos e animais. Curitiba: UFPR; São Carlos: EdUFScar. $446 \mathrm{pp}$.

Messias Basques

ARAUJO, Patrício Carneiro. 2017. Entre Ataques e Atabaques: intolerância religiosa e racismo nas escolas. São Paulo: Aché. 300 pp. Kelson Gérison Oliveira Chaves

Teixeira, Jacqueline Moraes. 2016. A mulher Universal: corpo, gênero e pedagogia da prosperidade. Rio de Janeiro: Mar de Idéias. $216 \mathrm{pp}$.

Andréia Vicente da Silva

\section{Instruções editoriais para os autores}

\section{Diretrizes para autores}




\section{N D E X}

CAMPOS V.19 N.1 jan.jun.2018

02

09

17

87

113

Apresentação

Dossier - Ecology and Feminism / Part I

Introduction to dossier Ecology and Feminism: political creations of indigenous women, quilombolas and peasants women

Fabiana Maizza e Suzane de Alencar Vieira

Feel the words, create trenches: women in defense of the Red de la vida

Luiza Dias Flores Damiana Bregalda Jaenisch

Noke Mevi Revôsho Shovima Awe: 'What is transformed by the tips o four hands' Nelly Barbosa Duarte Dollis

"The abode is a healer": the feminine as a force Luiza Dias Flores

Earth from the Bodies: Notes on Creativity in Works of Rosa Tisoy

Tatiana Lotierzo

The world of socio-environmental projects seen by indigenous women. Rethinking ecofeminism through the case of the Mêbêngôkre-Xikrin of the Terra Indígena Trincheira Bacajá (TITB, Pará, Brazil)

Stéphanie Tselouiko

\section{Articles}

Mana and Shamanic Substances: The reconfigurations of power among the siona of Colombia

Esther Jean Langdon 
Sea, river, mangrove. The spaces marked by the gender in the fishing communities of the state of Pará, Brazil

Christine Escallier

\section{Translations}

163

183

195

201

207

213

Tradition and knowledge, or outdoor living presentation to translation

Daniel Belik e Mariana Ciavatta Pantoja

Perceiving the Environment in Finnish Lapland Tim Ingold e Terhi Kurttila

\section{Informative Notes}

Yubaka Hayrá: Notes on the Indigenous Conference of Ayahuasca

Domingos Bueno da Silva

\section{Reviews}

BASINI, José. 2015. Indigenous in a country without indigenous. The aesthetics of disappearance: a study of indigenous images and ethnic versions. Manaus: Editora Travessia/Fapeam. 520pp. João Roberto Bort Júnior

BEVILAQUA, C.B.; VANDER VELDEN, F. (orgs.). 2016. Relatives, victims, subjects: anthropological perspectives on humananimal relations. Curitiba: UFPR; São Carlos: EdUFScar. 446 pp.

Messias Basques

ARAUJO, Patrício Carneiro. 2017. Between Attacks and Atabaques: religious intolerance and racism in schools. São Paulo: Aché. 300 pp. Kelson Gérison Oliveira Chaves

Teixeira, Jacqueline Moraes. 2016. The universal woman: body, gender and pedagogy of prosperity. Rio de Janeiro: Mar de Idéias. 216 pp.

Andréia Vicente da Silva

\section{Editorial Guidelines for Authors}

